\title{
Corpus
}

$8 \mid 2009$

Corpus de textes, textes en corpus

Les corpus au service d'une approche multidimensionnelle de certains faits de langue et de discours : les exemples de la concession et de l'apostrophe

\section{Michèle Monte}

\section{(2) OpenEdition \\ Journals}

Édition électronique

URL : http://journals.openedition.org/corpus/1689

ISSN : $1765-3126$

Éditeur

Bases; corpus et langage - UMR 6039

Édition imprimée

Date de publication : 15 novembre 2009

Pagination : 149-176

ISSN : 1638-9808

\section{Référence électronique}

Michèle Monte, « Les corpus au service d'une approche multidimensionnelle de certains faits de

langue et de discours : les exemples de la concession et de l'apostrophe », Corpus [En ligne], 8 | 2009, mis en ligne le 01 juillet 2010, consulté le 07 septembre 2020. URL : http://journals.openedition.org/ corpus/1689 


\title{
Les corpus au service d'une approche multidimensionnelle de certains faits de langue et de discours : les exemples de la concession et de l'apostrophe
}

\author{
Michèle MONTE \\ Université du Sud Toulon Var, Babel EA 2649
}

Cet article se propose d'examiner le rôle décisif du recours au corpus dans deux cas d'étude de marqueurs qui présentent une imbrication de valeurs sémantiques, textuelles et énonciatives nécessitant une étude fine des contextes. L'utilisation du corpus reste classique dans la mesure où il fournit un ensemble d'occurrences mais le travail de recherche resitue ces occurrences dans des contextes larges qui mobilisent parfois la connaissance entière du texte dont est issue l'occurrence. Ce n'est donc pas tant le travail sur corpus qui amène à reconsidérer le concept de texte, que la prise en compte du texte comme ensemble organisé, énonciativement et argumentativement cohérent, qui éclaire le fonctionnement de la langue. Je m'attacherai donc surtout à décrire l'activité interprétative et ses soubassements théoriques, d'autant plus nécessaire que les faits empiriques sont nombreux et apparemment divergents.

La première étude, collective, a porté sur les connecteurs concessifs et a fait l'objet d'un livre dirigé par Sylvie Mellet, Concession et dialogisme, publié en 2008. Menée par l'équipe «linguistique de l'énonciation et linguistique textuelle » du laboratoire Bases, Corpus, Langage, cette étude avait pour objectif de préciser le noyau sémantique de connecteurs concessifs adverbiaux souvent donnés pour synonymes: pourtant, pour autant, néanmoins, toutefois et cependant.

La deuxième étude, que j’ai menée seule, a porté sur les syntagmes en apostrophe, leur fonctionnement syntaxique, leur rôle textuel et pragmatique. Ayant commencé en 2005 par l'étude diachronique du marqueur $\hat{o}$ dans deux configurations 
différentes mais partiellement superposables, elle s'est poursuivie par une étude de l'apostrophe dans des genres littéraires différents et a donné lieu à la publication de plusieurs articles cités en bibliographie.

J'examinerai successivement les enjeux de l'approche sur corpus dans chacune des deux études puis je tenterai en conclusion de dégager les similitudes de ces démarches.

\section{L'étude des connecteurs concessifs ${ }^{1}$}

\subsection{La perspective diachronique}

Notre équipe étant constituée de spécialistes d'états de langue différents, depuis le XVIe siècle jusqu'à nos jours, nous souhaitions à la fois préciser la valeur de chaque connecteur et rendre compte de l'évolution diachronique du micro-système constitué par ces cinq adverbes. Pour mener à bien mener une étude sur les fréquences d'emploi des connecteurs sur cinq cents ans, le recours à une base de données telle que FRANTEXT s'avérait indispensable. Malgré les biais inhérents à FRANTEXT - présence de plusieurs éditions d'un même ouvrage, représentation inégale des différents genres selon les siècles considérés, nombre total de textes très variable d'un siècle à l'autre, classement générique parfois sujet à caution et nécessitant une vérification - la base de données nous a permis de dégager rapidement des résultats importants :

- le rôle central joué par toutefois pour le marquage de la concession au XVIe siècle et son déclin rapide à partir de la fin du XVIIe siècle ;

- l'émergence progressive de pourtant aux XVIe et XVIIIe siècles et son essor à partir de 1800, accompagné d'un fléchissement des autres connecteurs ;

- le parallélisme entre les courbes de fréquence de néanmoins et toutefois ;

- les écarts de fréquence d'un genre à l'autre ;

- les variations idiolectales ${ }^{2}$.

1 Les références de page sans autre précision se font dans cette partie à Concession et dialogisme, S. Mellet (dir.), 2008.

2 Pour chacun de ces points, je renvoie le lecteur à l'ouvrage lui-même. 
Les corpus au service d'une approche multidimensionnelle de certains faits de langue et de discours : concessifs et apostrophe

La valeur d'un connecteur, comme pour tout élément du système linguistique, étant dépendante de sa place dans le système, il était important de prendre en compte cette évolution globale du système pour interpréter correctement les résultats. Mais à cette première approche quantitative devait succéder une approche qualitative passant par l'examen des occurrences elles-mêmes et de leur contexte d'emploi.

Après un balayage exhaustif des occurrences de FRANTEXT, nous avons pour chaque siècle constitué un corpus échantillonné en sélectionnant pour chaque connecteur quelques auteurs représentatifs dans chaque grand genre littéraire (poésie, théâtre, romans et récits de voyage, correspondance, essais ou traités). Ces auteurs sont généralement ceux pour lesquels la fréquence relative du connecteur étudié est moyenne par rapport à l'ensemble des fréquences observées dans le siècle et le genre en question, ni trop élevée, ni trop faible. Mais nous avons parfois complété cet échantillon par les occurrences d'un auteur qui présentait des particularités d'emploi intéressantes, et dans le cas de pour autant, qui connaît une extension d'emploi récente, nous avons adjoint à cette collecte des exemples relevés dans la presse écrite et à la radio sans aucune prétention d'exhaustivité.

Nous avons donc disposé d'exemples nombreux, dotés grâce à FRANTEXT d'un contexte relativement limité, mais la sélection d'auteurs représentatifs à l'intérieur de chaque genre et siècle nous a permis par ailleurs de travailler sur des contextes plus larges en nous reportant aux œuvres intégrales et d'affiner la compréhension des enjeux pragmatiques présidant à l'emploi de tel ou tel connecteur. L'étude de l'interaction du connecteur avec son contexte a été en effet le principe directeur de notre travail.

\subsection{Le rôle déterminant du contexte}

Les études classiques sur les connecteurs (cf. par exemple Gettrup et Nolke 1984, Roulet et al. 1985, Anscombre 1983 et 2002) consistent à déterminer les propriétés des connecteurs en procédant par permutation sur des exemples en nombre réduit, qu'ils soient fabriqués ou attestés, et en portant des jugements d'acceptabilité sur des énoncés ou des enchaînements 
d'énoncés. Or, dans le cas qui nous occupe, une telle méthode s'avère impropre à définir réellement le noyau sémantique spécifique de chaque connecteur, les manipulations conduisant très rapidement à un brouillage et une incertitude radicale des jugements métalinguistiques.

Notre parti pris a consisté, au contraire, à rassembler un très grand nombre d'exemples attestés et à observer le contexte de ces exemples, en partant de l'hypothèse de travail suivante : le connecteur est un des éléments d'un contexte multidimensionnel dont les différents éléments s'influencent mutuellement.

Par exemple, la présence d'un modalisateur tel que certes ou un conditionnel dans l'énoncé $p$ situé en amont du connecteur est le signe d'une opération énonciative (distanciation du locuteur principal $\mathrm{L}$ par rapport à un point de vue rattaché à un locuteur second $\mathrm{l}_{2}$ ) qui entraîne le choix préférentiel d'un connecteur présentant la relation concessive comme programmée dès l'énoncé de $p$. A l'inverse, le fait que la proposition $q$ située en aval du connecteur s'insère dans une rallonge syntaxique telle qu'une relative appositive, un syntagme prépositionnel ou une subordonnée hypothétique indique que la relation concessive s'est construite au fil du discours et prédispose à l'emploi d'un connecteur apte à porter cette spécificité. Dès lors c'est l'étude minutieuse d'un grand nombre de contextes qui pourra nous mettre sur la voie du noyau sémantique propre à une unité, sans éliminer l'existence de zones frontières où deux connecteurs différents peuvent tous deux s'employer avec une égale pertinence.

A cet égard, le recours à des textes écrits littéraires ou scientifiques s'avère un avantage par rapport aux corpus oraux dans la mesure où le contexte s'y limite à la représentation discursive construite par le texte lui-même et où les énoncés sont à interpréter en s'appuyant uniquement sur ce contexte. Encore faut-il pouvoir déterminer ce qui, dans le contexte, témoigne d'opérations énonciatives susceptibles d'influencer le choix du connecteur. 
Les corpus au service d'une approche multidimensionnelle de certains faits de langue et de discours : concessifs et apostrophe

\subsection{Interroger le contexte : le choix des paramètres}

Le choix des observables au sein de ce contexte s'avérant capital, l'élaboration de la liste des paramètres à prendre compte a requis toute notre attention et a conjugué empirie et théorie. L'empirie a par exemple présidé à l'établissement de la liste des paramètres syntaxiques, à partir de l'étude préalable d'un sous-ensemble restreint d'occurrences afin de disposer d'un éventail de situations possibles dont je ne donnerai ici que quelques exemples ${ }^{3}$ :

- $\quad p$ et $q$ sont deux propositions indépendantes ;

- $\quad p$ et $q$ sont deux syntagmes de même nature, coordonnés ou juxtaposés (deux adverbes, deux adjectifs, deux verbes, etc.) ;

- $\quad$ q est une subordonnée hypothétique ou restrictive ;

- CONN-q est immédiatement précédé d’un autre connecteur (mais, et, etc.).

En ce qui concerne les paramètres lexico-sémantiques, les premiers relevés sur néanmoins ont montré que $p$ et $q$ contenaient souvent deux termes en opposition paradigmatique et l'étude du sous-corpus Poincaré montrait qu'avec toutefois, $p$ exprimait souvent une loi générale. Cela nous a incitées à nous appuyer sur l'opposition culiolienne entre détermination qualitative et quantitative et sur la notion de repérage pour élaborer d'autres paramètres dont nous avons ensuite vérifié la pertinence :

- $\quad p$ contient un superlatif ou toute autre forme d'intensif ;

- $\quad p$ contient un quantifieur ou tout autre marqueur qui est la trace d'une opération de parcours sur une classe d'occurrences ;

- $q$ contient un terme récapitulatif, synonyme ou hyperonyme, anaphorique d'éléments évoqués en $p$.

$\mathrm{Si}$ le choix des paramètres lexico-sémantiques doit beaucoup à la Théorie des Opérations Enonciatives, celui des paramètres argumentatifs est grandement redevable à la théorie de l'argumentation dans la langue dans la mesure où celle-ci s'attache à définir les actes illocutoires inscrits dans les

3 Pour une liste complète des paramètres, se reporter aux pp.13-15 de Concession et dialogisme. 
énoncés, à les rattacher à des énonciateurs et à évaluer leur poids respectif et la façon dont ils interagissent. Nous avons ainsi dégagé 18 paramètres relatifs à :

- la nature des actes illocutoires effectués en $p$ et $q$ (négation, exhortation, interrogation) ;

- la possibilité pour la suite du texte d'enchaîner tantôt sur $p$, tantôt sur $q$, tantôt sur leur synthèse ;

- la portée de l'acte effectué en $q$ qui, tantôt remet en cause une inférence de $p$, tantôt introduit une exception ou une limite à la validité de $p$, tantôt remet carrément en question le contenu ou la pertinence énonciative de p.

On voit que, dans cette liste de paramètres, ce qui compte, ce n'est pas tant la nature du raisonnement mis en œuvre que les actes effectués lors de l'énonciation de $p$ puis de q. Après avoir étudié avec soin l'opposition entre concession causale et concession argumentative que l'on trouve par exemple chez Moeschler 1989, ou celle qui distingue concession et adversation (Gettrup et Nolke 1984), nous sommes en effet arrivées à la conclusion que ces réflexions logico-philosophiques sur la concession, sans être inutiles pour comprendre la démarche d'un locuteur donné, ne peuvent permettre de classer les cinq connecteurs étudiés de façon satisfaisante car elles sont trop globales.

Sur le plan énonciatif, nous avons pris en compte le dialogisme inhérent à tout discours (Bres et Nowakowska 2006). Nous avons accordé un intérêt tout particulier au critère des tours de parole : il n'est en effet pas indifférent que $p$ et $q$ soient assertés par le même locuteur ou au contraire distribués sur les tours de parole de deux locuteurs différents. Mais la plupart des textes de notre corpus étant monologaux, nous avons aussi pris en compte la possibilité que $p$ représente, au sein du discours du locuteur unique, le discours d'un locuteur autre (dialogisme interdiscursif) ou que $p$ et $q$ correspondent à deux points de vue successifs du locuteur unique (autodialogisme). Enfin nous avons tenu le plus grand compte de la possibilité qu'offre la langue au locuteur de moduler son positionnement vis-à-vis des énoncés qu'il asserte: tous les phénomènes de modalisation et la possibilité que ces 
Les corpus au service d'une approche multidimensionnelle de certains faits de langue et de discours : concessifs et apostrophe

modalisateurs figurent soit en $p$ soit en $q$ ont pris place dans notre série de paramètres.

On voit donc que notre traitement du corpus repose sur des choix théoriques explicites privilégiant l'observation des traces de l'activité interlocutive, mais il faut ajouter aussi que c'est le côté peu opératoire des théories logiques de la concession qui nous a conduites à accorder la prééminence aux paramètres proprement linguistiques. Il semble bien en effet que ce qui est matérialisé par l'emploi d'un connecteur relève non pas d'un certain type de raisonnement logique mais plutôt de la façon dont le locuteur structure un domaine notionnel puis choisit un certain repérage énonciatif permettant de positionner les deux énoncés $p$ et $q$ l'un vis-à-vis de l'autre.

Par ailleurs, il est important de souligner que, dans notre liste de paramètres, le lexique et la syntaxe ne sont nullement minorés par rapport aux faits énonciatifs et aux actes de langage. Un tel choix est un des effets à mon sens très positifs du travail sur corpus. Celui-ci, en nous amenant à disposer pour chaque connecteur de plusieurs centaines d'exemples attestés, nous confronte à une très grande variété de situations syntaxiques ainsi que d'univers discursifs, ce qui nous conduit nécessairement à chercher des régularités permettant de traiter cette richesse référentielle et linguistique. L'établissement de paramètres ressortissant au lexique et à la syntaxe permet d'observer les données selon une grille qui ne privilégie ni le tout énonciatif ni le tout argumentatif, contrairement à d'autres travaux sur les connecteurs, et qui conduit progressivement à définir pour chaque connecteur un signifié abstrait dont les effets se manifestent sur tous les plans du discours à la fois.

C’est ainsi que néanmoins peut se définir tout d'abord sur le plan sémantique comme un connecteur opérant une inversion de gradient dans un domaine notionnel organisé sur le plan qualitatif entre deux pôles d'une même notion : l'énoncé $q$ est repéré par rapport à un premier énoncé $p$ " par identification de l'intensité de deux déterminations qualitatives appartenant à deux échelles d'orientation inverses (type : des engouements éphémères et néanmoins puissants) » (p. 9). La présence de termes qualitatifs en opposition paradigmatique en $p$ et $q$, ainsi 
que de superlatifs ou autres marqueurs d'intensité nous a orientées vers ce noyau sémantique, de même que la propriété syntaxique qu'a néanmoins d'opposer deux éléments infrapropositionnels (adjectifs notamment) de même nature. Cette spécificité sémantique s'articule à une spécificité argumentative qui consiste à mettre sur le même plan les deux termes en opposition sans hiérarchiser les points de vue: néanmoins indique qu'est invalidée une inférence que l'allocutaire aurait pu tirer de $p$ mais que l'opinion exprimée en $p$ reste valable. L'apparente contradiction est pleinement assumée par le locuteur, souvent anticipée dès l'énoncé de $p$ par des modalisateurs tels que certes ou sans doute ou par le fait que $p$ est une subordonnée concessive-restrictive : on a donc affaire à un dialogisme interne où le locuteur assume la vérité de $p$ sans prendre en charge les conséquences qui en découlent normalement et construit une unité énonciative supérieure à l'énoncé. On voit par cet exemple comment notre description du connecteur prend en compte les différents plans d'analyse et dérive les propriétés argumentatives et énonciatives du noyau sémantique que l'étude de corpus nous a permis de dégager.

Le recours au corpus, en nous donnant accès facilement à de nombreux exemples largement contextualisés, permet aussi de ne pas séparer le rôle textuel et le rôle argumentatif du connecteur: notre analyse de cependant propose une vision unitaire de l'adverbe où son rôle d'organisateur textuel introduisant un nouveau personnage, une nouvelle étape du texte, un nouveau point de vue narratif, et son rôle argumentatif consistant à mettre sur un pied d'égalité deux situations ou deux propriétés contradictoires s'originent dans sa spécificité sémantique, qui n'est pas concessive mais qui réside dans l'affirmation de l'existence simultanée de deux points de vue plus ou moins divergents. C'est pourquoi le rôle concessif de cependant, qui se développe au fil des siècles, a besoin d'être soutenu contextuellement par des oppositions modales en $p$ et $q$ ou par l'exhibition, via des marqueurs métadiscursifs ${ }^{4}$, d'un dialogisme interlocutif ou interdiscursif explicite.

4 Citons deux exemples empruntés l'un à Lipovetsky, l'autre à Lesourd et Gérard : «A cette interprétation qui a le mérite [...] il faut cependant 
Les corpus au service d'une approche multidimensionnelle de certains faits de langue et de discours : concessifs et apostrophe

\subsection{Jeu des paramètres : spécificité et malléabilité des connecteurs}

L'étude systématique pour chaque occurrence des nombreux paramètres que nous avons élaborés nous amène ainsi à dégager l'invariant de chaque adverbe, et pour les trois dont les fréquences sont voisines, à en dresser une carte d'identité assez précise qui montre bien qu'il n'y a pas synonymie. Si l'on résume dans un tableau les traits les plus marquants, on constate que sur chaque trait, deux des connecteurs s'opposent au troisième, et que chacun possède une configuration spécifique :

\begin{tabular}{|l|c|c|c|}
\hline & néanmoins & toutefois & cependant \\
\hline $\begin{array}{l}\text { égalité des 2 pdv : } \\
\text { n'est pas remis en } \\
\text { cause }\end{array}$ & $\mathrm{X}$ & & $\mathrm{X}$ \\
\hline $\begin{array}{l}\text { supériorité de q qui } \\
\text { vient limiter la validité } \\
\text { de } p\end{array}$ & & $\mathrm{X}$ & \\
\hline $\begin{array}{l}\text { concession } \\
\text { programmée dès } p\end{array}$ & $\mathrm{X}$ & & \\
\hline $\begin{array}{l}\text { concession construite } \\
\text { en discours }\end{array}$ & & $\mathrm{X}$ & $\mathrm{X}$ \\
\hline $\begin{array}{l}\text { maintien du cadre } \\
\text { discursif initial }\end{array}$ & $\mathrm{X}$ & $\mathrm{X}$ & \\
\hline $\begin{array}{l}\text { sortie hors du cadre } \\
\text { discursif initial }\end{array}$ & & & $\mathrm{X}$ \\
\hline
\end{tabular}

Mais si notre travail apporte de l'eau au moulin des linguistes qui récusent la possibilité en synchronie d'une totale synonymie entre deux lexèmes, il éclaire aussi un phénomène qu'on ne peut balayer d'un revers de plume et qui est celui du recouvrement partiel des zones d'emploi de ces connecteurs. En effet les multiples paramètres pris en compte ne convergent pas forcément tous dans le même sens, ce qui peut autoriser

objecter que $[. .$.$] »; « Cependant on peut suivre M. Jouffroy quand il$ nous montre que, à côté des mouvements de population, il y a eu aussi une stabilisation de celle-ci ». 
l'emploi de l'un ou l'autre des connecteurs en fonction du paramètre qui sera senti comme plus décisif par le locuteur. Ainsi, lorsque coexistent dans un même énoncé l'indication d'un parcours et celle d'une intensité, le locuteur a le choix entre néanmoins et toutefois et le contexte indique bien souvent pour quelle raison l'un des deux a eu sa préférence (p. 68).

Cette indifférence à tel ou tel trait du contexte est à notre avis un des facteurs qui peuvent expliquer l'extension de pourtant au fil du temps : en effet, sur le plan sémantique, ce connecteur ne semble pas sensible à l'opposition quantité / qualité (p. 112) et, sur le plan argumentatif, il s'accommode aussi bien de la remise en cause d'une inférence de $p$ (type le plus fréquent de concession, tous connecteurs confondus), que de celle de la validité de $p$, voire de son énonciation (pp. 113-115). Les paramètres discriminant les autres connecteurs se révélant décevants dans le cas de pourtant, qui, cela a été maintes fois souligné, peut accompagner aussi bien l'énoncé concédé $p$ que l'énoncé asserté $q$, nous avons pris acte de sa plasticité et avons essayé de comprendre ce qu'il y avait de commun à ces emplois apparemment si divers. C'est un paramètre syntaxique qui nous a alors aiguillées sur la bonne voie: le fait que pourtant apparaisse souvent dans une proposition principale précédée d'une subordonnée concessive en bien que ou quoique. Loin d'être pléonastique, ce pourtant nous est apparu comme ayant pour fonction de renforcer la proposition dans laquelle il se trouve et nous avons fait l'hypothèse que, dans tous ses emplois, pourtant indiquait l'affaiblissement de la proposition concédée (cf. pp. 117-122 pour le détail de la démonstration), affaiblissement pouvant aller jusqu'à la réfutation complète.

Plus généralement, le recours à des corpus pour la collecte de très nombreuses occurrences contextualisées joint à l'hypothèse théorique qu'un connecteur possède une valeur sémantique propre présente dans tous ses emplois, hypothèse monosémique qui s'oppose à l'idée de sens dérivés plus ou moins éloignés d'un sens premier, amène, me semble-t-il, les linguistes à une nécessaire inventivité explicative. En effet, ces deux contraintes de la recherche jouent apparemment en sens inverse: les occurrences nous confrontent, au-delà de la 
Les corpus au service d'une approche multidimensionnelle de certains faits de langue et de discours : concessifs et apostrophe

diversité du discours, à la variabilité de la langue, cependant que l'hypothèse monosémique nous oblige à ne pas nous en tenir à un classement superficiel de cette apparente diversité et à chercher la " forme schématique abstraite, stable et plastique à la fois » (p.8) qui puisse être fédératrice de tous ces emplois. Dans cette quête, la morphologie du connecteur, mot composé progressivement soudé, est une aide précieuse qui donne à voir comment l'expression de la concession s'appuie sur des marqueurs originellement destinés à signaler une concomitance, une variation proportionnelle, une comparaison sur une échelle d'intensité, une causalité. Mais surtout la double pression de l'hypothèse théorique et des variations contextuelles permet d'éviter l'écueil de l'empirie impuissante à dégager un fonctionnement abstrait commun aux différentes occurrences, et celui de la théorie trop peu en prise sur les réalisations effectives en discours.

Dans cette analyse, le contexte est défini comme un faisceau d'unités linguistiques en interaction les unes avec les autres. Le cadre pragmatique de l'interaction est pris en compte pour comprendre le choix de tel ou tel connecteur (cf. pp. 231232), mais la valeur sémantique spécifique du connecteur n’est pas dépendante de facteurs pragmatiques, par définition toujours mouvants. Nous avons pu montrer, en effet, qu'un même connecteur, toutefois en l'occurrence, souvent senti comme plus polémique qu'un autre, apparaît en réalité dans des interactions très variées où il pourra accompagner aussi bien des actes illocutoires qui ménagent les faces de l'interlocuteur (atténuation du point de vue du locuteur, limitation des inférences d'un énoncé de l'allocutaire mais préservation de sa validité) que des actes plus polémiques et plus dangereux pour l'interlocuteur (disqualification du point de vue de l'allocutaire ou de son énonciation). Ces variations pragmatiques s'originent dans une valeur commune qui, elle, est linguistique: la dissociation des points de vue assertés en $p$ puis en $q$, et l'interruption du mouvement discursif déployé en $p$.

Dans ce travail sur les connecteurs, la démarche inductive favorisée par le corpus s'est révélée véritablement heuristique puisqu'elle a abouti à différencier les adverbes concessifs en dégageant pour chacun d'eux une forme 
schématique abstraite qui lui confère une place à part au sein du micro-système dans un état de langue donné. D’autre part la diversité des paramètres nous a permis de comprendre comment le système pouvait évoluer et se réorganiser au fil du temps en marquant plus nettement tel ou tel type de relation concessive grâce à l'émergence d'un nouveau connecteur. Voyons à présent le rôle qu'a joué le corpus dans le travail sur l'apostrophe.

\section{L'étude de l'apostrophe}

L'apostrophe est une opération énonciative consistant à inscrire dans l'énoncé un SN détaché référant à l'allocutaire de l'énoncé: ce $\mathrm{SN}$ a les propriétés d'être indépendant des relations argumentales organisées par le verbe et non incident à un des SN de la phrase. Son expression est toujours facultative. En poésie, il est souvent précédé de ô. De cet ensemble de traits découle un programme de travail : identifier le rôle joué par $\hat{o}$, comprendre ce qui motive l'expression du SN vocatif dans certains énoncés et pas dans d'autres, repérer les zones de chevauchement avec d'autres constructions détachées et voir comment l'ambiguïté peut être levée ou au contraire comment s'instaure une sous-détermination. Seule l'étude du morphème ô peut bénéficier de l'aide que constituent des bases de données informatisées comme FRANTEXT. Pour le reste, le corpus ne peut être constitué que manuellement: une recherche sur les termes d'adresse, utilisés préférentiellement mais non exclusivement dans les SN en apostrophe, conduirait en effet à devoir éliminer après coup tous les emplois de ces termes termes de parenté, titres, profession - dans des SN intégrés à la phrase et le gain initial serait vite effacé par le tri à faire au sein des occurrences. Souhaitant travailler sur l'apostrophe, j'ai donc tout naturellement commencé par constituer un corpus diachronique des syntagmes $\hat{o}+\mathrm{SN}$ en poésie en m'aidant de FRANTEXT. 
Les corpus au service d'une approche multidimensionnelle de certains faits de langue et de discours : concessifs et apostrophe

\section{1. ô $+\mathrm{SN}$ : une ambiguïté marginale mais révélatrice ${ }^{5}$}

Le morphème $o$ peut introduire soit un SN exclamatif (personne 3) soit un SN en apostrophe (personne 2 ou 5). Cette ambiguïté potentielle peut être levée en discours par des facteurs syntaxiques ou lexicaux ou être au contraire entretenue, dans des conditions qu'il s'agit de préciser. D’autre part, ô est ressenti par l'informateur spontané comme archaïsant et lié à une langue littéraire qualifiée de "poétique». L’étude de corpus devait m'amener d'une part à préciser les facteurs d'ambiguïté, d'autre part à étudier diachroniquement l'usage du marqueur, afin de déterminer a) si le double usage de ô était stable à travers le temps ; b) si ô était effectivement plus utilisé dans les siècles passés qu'au XXe siècle. Sur le corpus FRANTEXT étiqueté " poésie », j’ai eu tout d'abord la surprise de constater que, rapportée au nombre total de mots des textes sélectionnés, la fréquence de ô augmentait au fil du temps. Partant de cette constatation, j'ai constitué un corpus diachronique regroupant des textes d'auteurs caractérisés par des fréquences moyennes, comprises entre 790 et 1871 millionièmes, à l'exception de deux auteurs (Apollinaire et Saint-John Perse) aux fréquences supérieures à 4000 millionièmes. Pour chaque auteur, j’ai constitué un sous-corpus de taille réduite afin de disposer d'un nombre comparable d'occurrences et de pouvoir les analyser en détail. J'ai retenu entre 40 et 60 occurrences par auteur en sélectionnant :

- la totalité des Antiquités de Rome et des Regrets de du Bellay (édition de 1558) ;

- les livres V, VI et VII des Tragiques de d'Aubigné (édition de 1623) ;

- les parties VIII et IX de Jocelyn de Lamartine (édition de 1836) ;

- le livre VI jusqu'au poème XXIII inclus des Contemplations de Victor Hugo (édition de 1856) ;

- la quatrième ode des Cinq grandes odes de Claudel (1910) ;

5 Les références de page sans autre précision se font dans cette sous-partie à Monte (2005). 


\section{MONTE}

- la totalité du Bestiaire et de Alcools d'Apollinaire (1911 et 1913) ;

- $\quad$ la totalité de Vents de Saint-John Perse (1946) ;

- la totalité du Roman inachevé d’Aragon (1956). Je disposais donc au total de 391 occurrences que j'ai resituées dans le contexte global de l'œuvre. J'ai tout d'abord observé une grande stabilité diachronique dans les emplois de ô :

- dès le XVIe siècle les emplois vocatif et exclamatif coexistent et leur proportion respective tient à la nature du texte et non à l'époque ;

- $\quad \hat{o}+\mathrm{SN}$ est adjoint à des énoncés aussi bien assertifs qu'interrogatifs ou injonctifs ;

- les 4 positions possibles du syntagme $\hat{o}+\mathrm{SN}$ - position initiale, en incise, finale et en énoncé nominal indépendant - s'observent chez tous les auteurs recourant à une délimitation graphique des énoncés, avec chez tous une nette prédilection pour la position initiale.

Toutefois deux différences diachroniques intéressantes sont apparues :

- la quasi disparition après le XVIIIe siècle des séquences $\hat{o}$ quel, $\hat{o}$ que et $\hat{o}$ combien, bien attestées chez du Bellay et d'Aubigné, et leur remplacement par des séquences oh quel, oh que et oh combien ;

- l'émergence tardive (sporadiquement au XIXe siècle, mais les travaux de Prat (1989) et Détrie (2006) signalent le même fait chez Baudelaire - et plus fréquemment au XXe siècle) d'emplois ambigus où lecture vocative et exclamative des syntagmes sont toutes deux possibles.

Il est intéressant d'observer que ces deux évolutions jouent en sens inverse : en effet, l'apparition de oh devant les marqueurs exclamatifs correspond à une tendance constatée par Y. Grinshpun (2005) et de plus en plus marquée au fil du temps à réserver $\hat{o}$ aux vocatifs et $o h$ à un emploi en contexte exclamatif : face à deux marqueurs homophones, la langue tend à spécialiser chacun d'eux dans une configuration syntaxique spécifique, de même que, sur le plan sémantique, les connecteurs tendent à se spécialiser pour éviter la synonymie. 
Les corpus au service d'une approche multidimensionnelle de certains faits de langue et de discours : concessifs et apostrophe

Mais, à l'inverse, l'apparition de syntagmes $\hat{o}+\mathrm{SN}$ ambigus contribue à un brouillage de la distinction entre les deux actes de langage que sont l'exclamation et l'apostrophe.

Forte de ces premières observations, il m'appartenait de poursuivre ma recherche dans deux directions : d'une part, il s'agissait de comprendre ce qui permettait l'ambiguïté de certaines configurations et éventuellement de relier leur émergence à d'autres phénomènes caractéristiques des mêmes corpus, d'autre part, il fallait essayer de mieux cerner le signifié de ô pour comprendre pourquoi c'était lui et non oh qui avait été progressivement sélectionné par la langue devant les SN vocatifs.

La première direction m'a conduite à montrer que, pour être ambigus, les syntagmes $\hat{o}+\mathrm{SN}$ doivent remplir deux conditions :

- emploi absolu ou absence de coréférence avec un élément de l'énoncé de personne 2 ou 5 (pronom, déterminant possessif) si le syntagme est adjoint à un énoncé verbal ;

- $\quad$ signifié du SN propice à l'invocation, soit à cause du sens inhérent au lexème, soit en raison de la situation d'énonciation et de l'idiolecte de l'auteur, qui peut, par exemple, avoir pour habitude d'invoquer des entités abstraites telles qu'un sentiment ou une sensation.

Si ces deux conditions sont satisfaites, on se trouve alors face à des énoncés sous-déterminés qui pourront recevoir l'une des deux interprétations par la mise en jeu de facteurs contextuels venant lever l'indécision ou cumuler les deux, le récepteur ayant l'impression que le locuteur à la fois invoque l'élément décrit par le $\mathrm{SN}$ et s'exclame à son propos. Cette situation est caractéristique de scénographies ${ }^{6}$ instables propres à la modernité poétique, dont on sait qu'elle a commencé précisément avec Baudelaire. Ces scénographies se caractérisent par de fréquents glissements de l'allocutif au délocutif (Charaudeau 1992) et vice-versa, d'autant plus aisés que la situation d'énonciation en poésie ne repose pas toujours

6 Sur cette notion propre à l'analyse de discours, cf. Maingueneau (2005 : 190-194). 
sur la mimésis d'échanges ordinaires, mais crée volontiers ex nihilo des dialogues avec des entités inanimées ou surnaturelles brusquement convoquées sur la scène énonciative à titre d'interlocuteurs du poète. De telles scénographies restent cependant étroitement dépendantes d'un projet esthétique particulier, et si les œuvres du corpus dues à Apollinaire et Aragon offrent un nombre assez élevé de ces configurations, tel n'est pas le cas de celles de Claudel ou Saint-John Perse, où la distinction entre SN vocatif et exclamatif est généralement plus claire.

Dans les occurrences sous-déterminées que m'offrait le corpus, $\hat{o}$ apparaissait fréquemment comme un soutien à l'interprétation vocative. D'autre part, l'analyse des constructions de la forme SN1 ô SN2 - relativement fréquentes dans Alcools ${ }^{7}$ montrait que, dans ces constructions, $\hat{o}$ autonomise le deuxième syntagme par rapport au premier mais n'exclut nullement une lecture exclamative parenthétique où SN2 serait une apposition à SN1. Il fallait donc essayer de trouver un signifié pour $\hat{o}$ qui prenne en compte cette irréductible dualité syntaxique. Il fallait aussi proposer une hypothèse explicative compatible avec la présence spécifique de ô dans la poésie. C'est ici que l'étude attentive des contextes larges et de l'organisation générale des œuvres retenues est intervenue. Je me suis en effet rendu compte que les syntagmes $\hat{o}+\mathrm{SN}$ se situent souvent dans des zones charnières entre discours et récit (pp. 63-64) ou lors du passage d'un acte de langage dominant à un autre et semblent avoir pour fonction de réactiver le souvenir, de le rendre présent, tout en impliquant plus étroitement l'allocutaire à des moments clés du texte. Si le SN désigne un référent susceptible d'être apostrophé, ô soutient l'interprétation vocative en rendant le référent présent sur la scène d'énonciation, et si tel n'est pas le cas, ô construit un espace commun au locuteur et à l'allocutaire permettant une prise en charge conjointe de l'exclamation.

7 On peut citer le vers très connu de «Zone » : "Bergère ô tour Eiffel le troupeau des ponts bêle ce matin» et ceux de «La Chanson du malaimé » : «Mon beau navire ô ma mémoire / Avons-nous assez navigué / Dans une onde mauvaise à boire ». 
Les corpus au service d'une approche multidimensionnelle de certains faits de langue et de discours : concessifs et apostrophe

Une telle interprétation de ô comme élément déixisant construisant un espace énonciatif consensuel commun au locuteur et à l'allocutaire, rend compte à mon avis de la disparition de $\hat{o}$ au profit de oh dans des constructions exclamatives qui se distinguent des interrogatives introduites par les mêmes outils quel et combien par un repérage ne nécessitant pas l'appel à l'allocutaire ${ }^{8}$. Il y avait en effet une certaine contradiction à utiliser dans le même énoncé un morphème $\hat{o}$ marqueur de la déixis interpersonnelle - et pour lequel l'emploi en apostrophe prédomine chez tous les auteurs à l'exception de du Bellay - et un morphème qu- en emploi exclamatif recentrant l'énoncé sur le locuteur. Oh et $\hat{o}$ se spécialisent donc progressivement, le premier dans un emploi interjectif ou dans des emplois exclamatifs où il accompagne un énoncé indifféremment verbal ou nominal, le second dans un soutien à l'apostrophe et dans des emplois exclamatifs devant $\mathrm{SN}$ où il garde un sens déixisant qui permet ces glissements vers l'allocutif que j'ai étudiés en détail dans mon article.

Le rôle ainsi dévolu à ô évite d'en faire un simple marqueur de poéticité : une telle option a en effet ceci de gênant qu'elle n'explique pas pourquoi ce marqueur s'accommode aussi bien des contextes exclamatifs que des contextes vocatifs, et elle explique encore moins pourquoi, à mesure que la notion de langue poétique s'affaiblit, le rôle de ô s'accroît, comme l'indique l'étude diachronique. Faire en revanche de $\hat{o}$ un marqueur de déixis consensuelle (cf. Danon-Boileau 1992), outre que cela lui assigne un signifié précis, l'inscrit dans le paradigme des mots du discours chargés de gérer l'interaction et l'orientation égocentrée ou allocentrée du discours. Or il me semble toujours avantageux d'inscrire un phénomène propre à certains types de discours, ici le discours poétique, dans un ensemble plus vaste de phénomènes langagiers, afin de saisir le particulier au sein du général. Par ailleurs, voir dans ô un marqueur de déixis consensuelle rend bien compte du fait que dans tout le corpus étudié l'injonction assortie d'un $\hat{o}+\mathrm{SN}$ ait

8 On se souvient que c'est par un centrage sur l'attracteur que Culioli (1990 : 121-122) distingue l'exclamation de l'interrogation où le parcours ne se stabilise pas et impose dès lors le recours au co-énonciateur. 
toujours un sens de prière ou d'exhortation et jamais d'ordre ou d'interdiction. Elle pose l'allocutaire comme un allié potentiel, et non comme un ennemi (cf. p. 66). On comprend aussi que $\hat{o}$ soit très fréquent devant le mot « Dieu » si l'on considère que le fidèle doit d'abord créer un espace commun entre lui et son Dieu, et le convoquer en quelque sorte sur la scène énonciative avant de s'adresser à lui. Plus qu'une marque de déférence ou d'emphase à laquelle on l'assimile parfois, j'interprète donc $\hat{o}$ comme un outil de présentification qui garantit la présence et l'empathie de l'allocutaire ${ }^{9}$ aussi bien dans le cas des syntagmes vocatifs, que dans celui des énoncés exclamatifs. J'associe dès lors son expansion dans la poésie au fil des siècles à d'autres phénomènes montrant que la poésie investit à partir du XIXe siècle des formes de subjectivité où les pôles du je et du tu ne sont pas distingués, et que la théorie praxématique résume sous le terme de subjectivité en idem ${ }^{10}$.

Dans le cas de $\hat{o}+\mathrm{SN}$, l'étude d'un vaste corpus présentait de grands avantages : les cas d'ambiguïté entre les deux interprétations vocative et exclamative se révélant relativement rares, seul le fait de disposer de nombreuses occurrences permettait de définir avec assez de précision les conditions auxquelles une telle ambiguïté pouvait naître. Par ailleurs l'étude diachronique permettait à la fois de circonscrire temporellement ces occurrences ambiguës et d'en déceler les prémices dans des états de langue plus anciens où l'ambiguïté n'est d'ordinaire pas vue car on adopte systématiquement l'une des deux lectures en se fiant au contexte. Enfin seule la contextualisation large des occurrences pouvait mettre sur la voie d'un signifié de $\hat{o}$ à la fois assez précis et suffisamment abstrait pour rendre compte de tous les effets de sens en discours. Comme pour tous les marqueurs qui désignent non pas des référents mais des opérations ou des positionnements énonciatifs, l'étude contextuelle permet mieux que l'intuition ou la manipulation d'énoncés de préciser le rôle du marqueur et son interaction avec le contexte.

9 Je diverge sur ce point de Grinshpun (2008).

10 Pour plus de détails, on pourra lire J.-M. Barbéris (2002) ainsi que Monte (à paraître). 
Les corpus au service d'une approche multidimensionnelle de certains faits de langue et de discours : concessifs et apostrophe

Ce premier travail m'avait montré l'intérêt de travailler sur une question encore peu étudiée ${ }^{11}$, à savoir l'apostrophe, dans une perspective à la fois énonciative, syntaxique et textuelle. C'est à cette fin que j'ai constitué un nouveau corpus prenant en compte la question des genres.

\subsection{Corpus et spécificités génériques ${ }^{12}$}

Le corpus que j'ai constitué à cette occasion comprend des œuvres écrites entre 1880 et 1990 à l'exception des oraisons funèbres de Bossuet. J'aurais pu évidemment rechercher des oraisons funèbres du XXe siècle - Malraux en fournirait sans doute un bon exemple, mais l'idée ne m'en est pas venue à ce moment-là. Le recours à Bossuet et à Blum reposait en revanche sur le souhait mûrement réfléchi de confronter l'usage littéraire de l'apostrophe à son usage dans des discours travaillés mais s'inscrivant dans une situation d'énonciation non fictive. J'ai donc choisi des nouvelles de Maupassant et un roman d'Eugène Le Roy pour les récits littéraires, des pièces de Sartre, Genet et Koltès pour le théâtre, des recueils d'Apollinaire, Claudel, Aragon et Saint-John Perse pour la poésie, et j'ai comparées ces œuvres entre elles ainsi qu'au corpus de référence constitué de trois oraisons de Bossuet, de deux discours politiques de Léon Blum ainsi que de sa plaidoirie lors du procès de Riom. Cet échantillon n'a évidemment pas de valeur statistique, et des particularités idiolectales ont pu infliger un biais à mes observations, néanmoins les enseignements que j'en ai tirés et que je rappellerai brièvement me paraissent fournir des points de référence solides pour des travaux ultérieurs.

Je me suis d'abord préoccupée de la forme des syntagmes en apostrophe et mes relevés portant sur plusieurs centaines d'occurrences ont confirmé les conclusions de Lambrecht (1998). Ils ont mis en valeur l'extrême fréquence

11 Le travail de Catherine Détrie, De la non-personne à la personne: l'apostrophe nominale, publié en 2006 après le début de mes propres recherches, s'appuie lui aussi sur différents corpus : ses conclusions sont très semblables aux miennes.

12 Les deux articles de référence - Monte (2008) et (2009) - sont cités ici sous l'année suivie du numéro de page. 
des noms propres, des noms communs (sans déterminant ou précédés du possessif) indiquant un statut ou une relation, et des pronoms toniques. Parmi ces nombreuses occurrences, certaines se bornent à interpeller l'allocutaire en le désignant par un titre ou un nom qui ne font que confirmer ce que nous savons déjà de la personne ou de la relation qui l'unit au locuteur, alors que d'autres prédiquent à son égard une propriété nouvelle, axiologiquement marquée, et rentrent de ce fait dans la catégorie des insultes ou des hypocoristiques. La littérature à ce sujet a souvent isolé les insultes au sein des apostrophes au motif que les insultes bénéficieraient d’une complétude informationnelle et d'une valeur prédicative dont les autres apostrophes seraient dépourvues; or ce que montrent mes exemples, c'est que la ligne de partage entre apostrophes prédicatives et non prédicatives passe non pas par leur contenu sémantique mais par leur insertion ou non dans un énoncé-hôte. Insérée dans l'énoncé-hôte, l'apostrophe renvoie à du savoir préalable ou présenté comme tel, et vient justifier pragmatiquement l'acte de langage effectué au moyen de l'énoncé-hôte tout en réglant le positionnement des interactants. Indépendante, l'apostrophe est un énoncé qui superpose deux actes de langage :

- un acte de langage allocutif, de valeur illocutoire diverse (appel, reproche, insulte, etc.), indiqué par l'intonation qui fait d'un simple SN un énoncé adressé, à distinguer par exemple d'un énoncé averbal exclamatif ;

- un acte de prédication dont le thème est l'allocutaire présent dans la situation d'énonciation et dont le prédicat résulte de la combinaison dans l'apostrophe d'un signifié lexical et d'un intonème porteur de la valeur illocutoire.

Le travail sur corpus fait apparaître nettement que les valeurs illocutoires sont construites à réception par la combinaison entre le signifié du lexème et l'intonation, reconstituée à l'écrit en fonction des indications contextuelles. En effet, on trouve au sein d'une même œuvre des occurrences d'un même terme d'adresse dans des contextes très différents imposant des interprétations, et donc, si le texte est oralisé, des 
Les corpus au service d'une approche multidimensionnelle de certains faits de langue et de discours : concessifs et apostrophe

intonations bien différentes elles aussi. Par ailleurs, on se rend compte que l'apostrophe insérée dans l'énoncé-hôte, même lorsqu'elle a un fort contenu axiologique, ne vient qu'en appui à l'acte de langage porté par l'énoncé et reste secondaire (cf. 2009, pp. 392-394). Deux exemples illustreront ces résultats :

(1) Le malheureux criait comme un porc qu'on va saigner, ne s'interrompant que pour demander grâce d'une voix piteuse. «Allons, tais-toi, braillard! ne vois-tu pas tous les autres sur pied ?...» (Le Roy)

(2) CAL : Ne sois pas si pudique, petite bonniche. J'ai l'instinct moi, pour certaines choses! LEONE : Je nous trouve si laids ! [...] CAL : Pudique! LEONE : Bandit ! (Koltès)

En (1), le remplacement de «braillard » par un nom propre ne modifie que marginalement l'interaction : certes, l'atteinte à la face positive de l'allocutaire s'en trouve atténuée, et la cohésion textuelle amoindrie ${ }^{13}$, mais l'enjeu principal de l'énoncé reste l'injonction «tais-toi ». Il en est de même dans le premier énoncé de (2) pour "petite bonniche ». La suppression de ces apostrophes ne perturbe pas le fonctionnement illocutoire de l'énoncé. En revanche, «Pudique!» et «Bandit!» sont essentiels à l'évolution du dialogue. Mais la valeur de l'énoncé doit être soigneusement distinguée du signifié lexical, comme le montre l'emploi inattendu de «pudique» comme quasi insulte $^{14}$.

Cependant la distinction entre apostrophe indépendante et apostrophe intégrée dans un énoncé-hôte est peut-être appréhendable sans corpus par des manipulations sur énoncé. Là où le corpus s'avère indispensable, c'est pour appréhender le rôle textuel et énonciatif de l'apostrophe. Il n'est pas question bien entendu de reprendre ici tous les résultats exposés dans

13 Comme souvent dans les romans, l'apostrophe entretient un lien sémantique avec l'énoncé-hôte - elle justifie ici l'injonction -, mais aussi avec les actions relatées dans le récit enchâssant le discours rapporté.

14 Des recherches complémentaires sur corpus auraient pu faire apparaître symétriquement des cas où «bandit » est associé à une intonation méliorative. 
mon article de 2008. Je ne mentionnerai que quelques faits significatifs.

La fonction primordiale de l'apostrophe semble bien être de resserrer la relation entre locuteur et allocutaire au moment où sont effectués les actes illocutoires les plus importants et les plus sujets à controverse. Les observations convergent sur ce point quel que soit le genre considéré. Les textes narratifs littéraires et les discours non littéraires font apparaître également qu'elle joue un rôle important d'articulateur textuel lors des changements de parties. Le fait qu'on ne retrouve pas cette fonction dans les textes de théâtre étudiés tient à mon avis à l'absence de longues tirades où la division en parties aurait pu avoir un sens et au primat qui est donné dans ces textes à la gestion de la relation interlocutive. Mais il faudrait prolonger ce travail par une comparaison avec des corpus oraux pour vérifier si le rôle de structuration joué par l'apostrophe dans des textes écrits et des discours préalablement rédigés existe aussi dans l'oral spontané. Il faudrait aussi mener une étude sur de larges corpus narratifs pour comparer le rôle dévolu aux segments en apostrophe et celui que remplissent les énoncés introduisant ou escortant le discours rapporté en ce qui concerne la dénomination des interactants et l'explicitation de leur positionnement socio-affectif.

Concernant la valeur pragmatique de l'apostrophe, les travaux antérieurs ont surtout insisté sur son rôle dans l'adoucissement des actes menaçants pour les faces de l'allocutaire. Mais une telle hypothèse a l'inconvénient d'attribuer à une fonction syntactico-énonciative prévue par le système de la langue des valeurs étroitement dépendantes du contexte et susceptibles d'infinies nuances et contre-exemples. C'est ainsi que mon corpus m'offrait des emplois dans des contextes très consensuels, d'autres dans des cas de grande tension énonciative où l'apostrophe pouvait effectivement apporter un certain adoucissement, et d'autres au contraire où l'apostrophe, par son signifié dépréciatif, accentuait la tension, sans parler des cas où elle pouvait produire ou accompagner un revirement dans les relations interlocutives. Comme dans le cas des connecteurs, l'issue consistait à mon sens à rechercher une valeur abstraite suffisamment large pour rendre compte de ces 
Les corpus au service d'une approche multidimensionnelle de certains faits de langue et de discours : concessifs et apostrophe

différents effets en discours. Les travaux de M.-A. Morel et L. Danon-Boileau ainsi que ceux de J.-M. Barbéris m'invitaient à regarder si l'apostrophe se situait plutôt du côté d'une subjectivité consensuelle où les pôles des deux énonciateurs sont faiblement distingués, ou du côté d'une subjectivité marquant nettement l'altérité du je et du $t u$. Une fois posé en ces termes, le problème paraissait assez facile à résoudre : si le signifié du SN mis en apostrophe est souvent vecteur d'une certaine politesse rituelle, et parfois d'un sentiment d'affection, l'acte même de l'apostrophe doit être dissocié de ce signifié conjoncturel pour être envisagé de façon plus abstraite comme un souci du locuteur de se positionner comme distinct de son allocutaire. Il arrive ainsi que l'apostrophe rentre dans une stratégie discursive quelque peu duplice où le locuteur n'inscrit son allocutaire dans le discours que pour mieux affirmer sa propre position ou pour mieux lui faire admettre ses vues. D'autres fois, au contraire, l'apostrophe sert de point d'appui à un acte où s'affirment la communauté de vues, la complicité des deux interlocuteurs, mais cette complicité passe par la reconnaissance de leurs identités spécifiques et non par une fusion des instances.

Le dernier enseignement de cette étude sur corpus concerne la spécificité relative de la poésie au sein des autres genres littéraires. Cette spécificité de la poésie concerne tout d'abord la scène d'énonciation, ainsi que je l'ai dit plus haut : alors que le théâtre, le récit - et d'ailleurs la poésie narrative illustrée par exemple par Jocelyn de Lamartine - imitent les échanges ordinaires, la poésie lyrique se donne beaucoup de liberté en donnant au locuteur identifié au poète de nombreux allocutaires et en le montrant en train d'interpeller des morts, des êtres inanimés, des concepts, etc. Je fais l'hypothèse que c'est pour cette raison qu'on y observe aussi fréquemment des glissements de l'allocutif au délocutif et vice-versa : l'étude de $\hat{o}+\mathrm{SN}$ m'avait confrontée crucialement à ce phénomène, mais l'étude suivante a confirmé qu'il concerne tous les syntagmes en apostrophe et pas seulement ceux qui sont précédés de $\hat{o}$.

Mais la multiplicité des allocutaires et le caractère non réaliste des dispositifs énonciatifs entraînent d'autres conséquences : le rôle socio-discursif de l'apostrophe y passe 
au second plan derrière un rôle d'identification ${ }^{15}$ et, plus étonnant encore, de description des interactants. En effet, bien que la mise en apostrophe concerne habituellement des référents accessibles dans la mémoire discursive, la poésie ne se prive pas d'introduire pour la première fois en position d'apostrophe des référents jusqu'alors inconnus. L'émancipation par rapport au réalisme entraîne également dans certains cas l'apparition de longs SN enrichis de toutes sortes d'expansions (adjectifs, propositions relatives, compléments prépositionnels) ou accompagnés d'appositions. Ce phénomène, qui trouve ses origines dans ce qu'on appelle traditionnellement les épithètes homériques, semble caractéristique en poésie du XXe siècle de certains idiolectes (Apollinaire, Saint-John Perse) : il peut ainsi constituer un trait définitoire d'une poésie à tendance épique ou ancrée dans la mythologie. Quoi qu'il en soit de l'extension de ce phénomène, on constate donc que, dans certains poèmes, les apostrophes jouent un rôle non négligeable à la fois dans la constitution et l'évolution de la scénographie et dans la cohésion textuelle par l'introduction de nouveaux actants ou par leur réitération et leur redescription au fil du texte (cf. Monte 2007 pour un travail systématique de cette question sur le recueil Vents de Saint-John Perse). Mais ce rôle spécifique se rattache néanmoins au fonctionnement plus général de l'apostrophe appréciative qui, dans tous les genres, tantôt étaye l'énoncé dans lequel elle s'insère, tantôt est légitimée par cet énoncé même (Monte 2008: 1425-1426), dans un effet de boucle entre la dénomination choisie et l'acte illocutoire effectué par la profération de l'énoncé. Simplement, alors que les bénéfices de l'apostrophe appréciative sont en général de type argumentatif, en poésie ils sont surtout référentiels et textuels et contribuent à l'effet d'évocation théorisé par Dominicy (1992).

15 Ce rôle existe dans l'interaction ordinaire et dans les genres seconds lorsque la situation entraîne l'existence potentielle de plusieurs interlocuteurs qu'il faut alors discriminer. 
Les corpus au service d'une approche multidimensionnelle de certains faits de langue et de discours : concessifs et apostrophe

\section{Conclusion}

On aura constaté au fil de la lecture que, dans ces travaux, les textes inclus dans les corpus ne sont pas vraiment interrogés dans leur dimension matérielle ou leur clôture. En revanche, ils sont appréhendés dans leurs multiples dimensions : cohésion textuelle, prise en charge énonciative, orientation argumentative. Si cette étude ne fournit donc pas beaucoup d'éléments pour une réflexion sur une éventuelle redéfinition du texte, elle montre en revanche que la multidimensionnalité du texte est une aide à la compréhension des phénomènes linguistiques. Envisager les liens entre énoncés marqués par les connecteurs uniquement sur le plan argumentatif rend par exemple beaucoup plus difficile l'appréhension des spécificités d'un connecteur que si on prend aussi en compte le signifié lexical de certains éléments en $p$ et $q$ ou l'organisation syntaxique des deux énoncés. Le texte apparaît à cet égard comme une structure résultant de l'interrelation entre différents réseaux (cf. Adam 2005: 31) ayant chacun leur propre logique et dont le poids respectif peut varier dans la pesée inconsciente qu'opère le locuteur au moment de choisir par exemple entre néanmoins et toutefois, pourtant ou cependant. La syntaxe, avec les possibilités de détachements et de rallonges qu'elle offre, semble le lieu où les relations complexes entre l'à dire, le dire et le dit (Détrie, Siblot, Verine 2002: 22) deviennent fugitivement appréhendables, et dans des textes écrits soigneusement contrôlés, les ambiguïtés qui subsistent, les glissements de certains SN de la $3^{\text {ème }}$ à la $2^{\text {ème }}$ personne, les réévaluations que cela entraîne, sont le signe de ces rétroactions incessantes entre les trois instances distinguées par la praxématique.

L'approche sur corpus informatisés, en sélectionnant rapidement des occurrences au contexte limité, permet par la mise en série qu'elle opère de mieux embrasser cette structuration complexe, et met sur la voie d'hypothèses puissantes qui prennent en compte la variabilité de la langue sans se perdre dans l'apparente dispersion des phénomènes. Extrayant les occurrences des textes dont elles sont issues, le corpus les rend en quelque sorte à l'interdiscours auquel les 
textes appartiennent grâce à la mise en paradigme d'exemples issus d'auteurs et d'œuvres différentes appartenant au même genre. Ce faisant, l'approche sur corpus permet de vérifier l'impact des superstructures génériques sur le choix de tel ou tel lexème ou de telle ou telle structure syntaxique, mais tout aussi bien d'en montrer dans certains cas le poids relativement faible et l'universalité de certains fonctionnements. L'outil statistique met en évidence certaines singularités idiolectales mais remet aussi en question des idées reçues, comme on a pu le voir avec ô. Il prémunit ainsi l'observateur contre certaines intuitions mal fondées. Mais l'analyse de multiples occurrences de faible étendue doit être complétée par la recontextualisation d'au moins une partie d'entre elles dans la totalité textuelle auxquelles elles appartiennent, afin de pouvoir faire bénéficier l'analyse des observations relatives à la composition du texte et à sa prise en charge énonciative globale. Une telle démarche de va-et-vient entre le paradigme des occurrences et la continuité / discontinuité du texte où elles avaient été prélevées s'est avérée déterminante dans la compréhension du rôle de l'apostrophe ou du morphème $\hat{o}$, ou dans l'appréhension des spécificités argumentatives de cependant ${ }^{16}$. On voit par là que l'approche sur corpus à la fois relativise la notion de singularité textuelle en valorisant la famille de textes plutôt que telle réalisation particulière, et revalorise le texte comme totalité multidimensionnelle où les facteurs de cohésion et de cohérence peuvent être étudiés indépendamment mais ne prennent sens que dans leur mise en relation.

\section{Références bibliographiques}

Adam J.-M. (2005). La linguistique textuelle. Paris : Armand Colin.

Anscombre J.-C. (1983). « Pour autant, pourtant (et comment) : à petites causes grands effets ", Cahiers de linguistique française 5 : 37-85.

16 Un travail en cours sur le dialogisme des si $\mathrm{P}$ non conditionnelles a bénéficié de ce même parti pris méthodologique. 
Les corpus au service d'une approche multidimensionnelle de certains faits de langue et de discours : concessifs et apostrophe

Anscombre J.-C. (2002). «Mais / pourtant dans la contreargumentation directe: raisonnement, généricité et lexique », LINX 46 : 115-129.

Barbéris J.-M. (1998). «Identité, ipséité dans la deixis spatiale », L'Information grammaticale 77 : 28-32.

Barbéris J.-M. (2001). Articles "Subjectivité dans le langage », "Subjectivité en même vs en soi-même » in Détrie, Siblot et Verine (dir.).

Bres J. et Nowakowska A. (2006). «Dialogisme : du principe à la matérialité discursive ", in L. Perrin (éd.) Le sens et ses voix. Metz: Publications de l'Université Paul Verlaine, pp. 21-48.

Charaudeau P. (1992). Grammaire du sens et de l'expression. Paris : Hachette.

Danon-Boileau L. (1992). "Ce que “ça” veut dire: les enseignements de l'observation clinique ", in M.A. Morel et L. Danon-Boileau (éds.), La deixis. Paris : PUF, pp. 415425.

Détrie C. (2006). De la non-personne à la personne: l'apostrophe nominale. Paris : CNRS éditions.

Détrie C., Siblot,P. et Verine,B. (2001). Termes et concepts pour l'analyse du discours. Une approche praxématique. Paris : Champion.

Dominicy M. (1992). «Pour une théorie de l'énonciation poétique ", in W. de Mulder, F. Schuerewegen et L. Tasmowski (éds), Enonciation et parti-pris. Amsterdam : Rodopi, pp. 129-142.

Gettrup H. et Nølke H. (1984). "Stratégies concessives : une étude de six adverbes français ", Revue romane 19, 1 : 3-47.

Grinshpun Y. (2005). " $O$ et $o h$ : une graphie entre langue et discours », Marges linguistiques 9: 242-255, revue électronique http : //www.marges-linguistiques.com.

Grinshpun Y. (2008) Ô entre langue(s), discours et graphie. Paris /Gap : Ophrys.

Lambrecht K. (1998). " Sur la relation formelle et fonctionnelle entre topiques et vocatifs », Langues 1 : 34-45. 
Maingueneau D. (2005). Le discours littéraire. Paris : Armand Colin.

Mellet S. (dir.) (2008). Concession et dialogisme. Les connecteurs concessifs à l'épreuve des corpus. Berne: Peter Lang (coll. "Sciences pour la communication » $\left.\mathrm{n}^{\circ} 85\right)$.

Moeschler J. (1989). Modélisation du dialogue. Représentation de l'inférence argumentative. Paris : Hermès.

Monte M. (2005). « $\hat{O}+$ SN dans les textes poétiques : entre apostrophe et exclamation », Modèles linguistiques, tome XXVI-2, vol. 52 : 45-68.

Monte M. (2007). «Tension énonciative et cohésion textuelle dans Vents, Chronique et Chant pour un équinoxe : le rôle des vocatifs », in Laure Himy (éd.) Questions de style 4 : 61-79; revue électronique de l'Université de Caen http://www.unicaen.fr/services/puc/revues/thl/questionsdestyle/

Monte M. (2008). "Usages littéraires de l'apostrophe: fonctions textuelles et pragmatiques et spécificités génériques ", Actes du $1^{\text {er }}$ congrès mondial de linguistique française de juillet 2008 à Paris, CD-Rom, EDP Sciences, pp. 1405-1416.

Monte M. (2009). «L'apostrophe dans les textes littéraires : prédicativité et valeurs illocutoires ", in D. Apothéloz, B. Combettes et F. Neveu (éds), Les linguistiques $d u$ détachement. Berne : Peter Lang, pp. 389-400.

Monte M. (à paraître). «Variations de l'actualisation dans la poésie contemporaine », Journée d'étude «L'actualisation de l'intersubjectivité en discours » du 30 mars 2009 à l’Université Paul Valéry de Montpellier.

Morel M.A. et Danon-Boileau L. (1998). Grammaire de l'intonation. Paris / Gap : Ophrys.

Prat M-H. (1989). "L'apostrophe dans Les Fleurs du mal », L'Information grammaticale $40: 18-22$ et $41: 39-43$.

Roulet E. et al. (1985). L'articulation du discours en français contemporain. Berne : Peter Lang. 